\title{
BMJ Open Association between allostatic load and mortality among Chinese older adults: the Chinese Longitudinal Health and Longevity Study
}

Tianhang Zhang, ${ }^{1,2}$ Lijing L Yan, ${ }^{1,3}$ Hua-Shuai Chen, ${ }^{4}$ Hai-Yu Jin, ${ }^{1}$ Chenkai Wu (D) ${ }^{1}$

To cite: Zhang T, Yan LL, Chen $\mathrm{H}-\mathrm{S}$, et al. Association between allostatic load and mortality among Chinese older adults: the Chinese Longitudinal Health and Longevity Study. BMJ Open 2021;11:e045369. doi:10.1136/ bmjopen-2020-045369

- Prepublication history and additional online supplemental material for this paper are available online. To view these files, please visit the journal online. (http://dx.doi.org/10. 1136/bmjopen-2020-045369).

Received 01 October 2020 Accepted 14 July 2021

\section{Check for updates}

(c) Author(s) (or their employer(s)) 2021. Re-use permitted under CC BY-NC. No commercial re-use. See rights and permissions. Published by BMJ.

${ }^{1}$ Global Health Research Center, Duke Kunshan University, Kunshan, Jiangsu, China ${ }^{2}$ School of Public Health, Imperial College London, London, UK

${ }^{3}$ Duke Global Health Institute, Durham, North Carolina, USA ${ }^{4}$ Center for the Study of Aging and Human Development, Duke University, Durham, North Carolina, USA

\section{Correspondence to} Dr Chenkai Wu; chenkai.wu@dukekunshan. edu.cn

\section{ABSTRACT}

Background Allostatic load (AL) has shown that high burden of $A L$ is associated with increased risk of adverse outcomes, but little attention has been paid to China with largest ageing population in the world.

Objective This study is to examine the association between $\mathrm{AL}$ and all-cause mortality among Chinese adults aged at least 60 years.

Design Population-based prospective cohort study. Setting In 2011-2012, an ancillary study, in which a blood test was added, including a total of 2439 participants, was conducted in eight longevity areas in the Chinese Longitudinal Healthy Longevity Survey.

Participants The final analytical sample consisted of 1519 participants (mean \pm SD age: men $80.5 \pm 11.3$ years; women $90.2 \pm 11.8$ years and $53 \%$ women).

Primary outcome measure Cox models were used to examine the association between $\mathrm{AL}$ and mortality among men and women, separately. Analyses were also adjusted for potential confounders including age, ethnicity, education and marital status, smoking and exercise.

Results Male with a medium AL burden (score: 2-4) and high AL burden (score: $5-9$ ) had a $33 \%$ and $118 \%$ higher hazard of death, respectively, than those with a low AL burden (score: $0-1$ ). We did not find significant difference between females with different levels of $A L$ burden. Conclusion Higher AL burden was associated with increased all-cause mortality among Chinese men aged at least 60 years. However, we did not find strong association among women. In conclusion, Intervention programmes targeting modifiable components of the AL burden may help prolong lifespan for older adults, especially men, in China.

\section{BACKGROUND}

Allostatic load (AL) is conceptualised as the cumulative wear and tear on multiple physiological systems resulting from repeated adaptation to stressors. ${ }^{1-4}$ In the absence of a gold standard, many operational definitions of AL have been proposed. The most commonly used construct of AL was developed by Seeman et al who have used two categories of biomarkers for quantifying AL. ${ }^{45}$ The first category (called primary mediators) includes

\section{Strengths and limitations of this study}

- This is the first study to investigate the association between allostatic load (AL) and mortality using a Chinese population.

- The Chinese Longitudinal Healthy Longevity Survey dataset that is a large nationally representative old population survey in China.

- The updated quartile risk method for biomarkers body mass index, total cholesterol and triglyceride among older adults.

- Lack of primary neuroendocrine biomarkers such as cortisol in constructing the AL score, which may influence the finding presented.

- There is huge lost to follow-up (>20\%; 552 of 2439), although only 3-7 year range of follow-up (2011 to 2014-2018), which may underestimate the association.

biomarkers the body releases in response to stress, such as cortisol and dehydroepiandrosterone sulfate; the second category comprises secondary outcomes that result from the effects of primary mediators. Examples of biomarkers are blood pressure (BP), cholesterol and the waist-hip ratio. ${ }^{4}$

A number of studies identified that a high burden of AL is associated with increased risk of adverse outcomes including cardiovascular disease (CVD), functional decline and mortality among the older adults. ${ }^{126-10}$ For example, in a 7-year longitudinal study conducted in 2006, increased AL score was associated with higher mortality among older population. ${ }^{8}$ In a cohort study of 1023 community-dwelling older adults in Taiwan, researchers found that higher AL score was related with higher death rate. ${ }^{7}$ Additionally, some studies found that women and men experienced chronic stress in different ways. For example, Yang and Kozloski revealed gender differences in the AL biomarkers and the age trajectories of physiological dysregulation. ${ }^{11}$ Women had a higher level 
of inflammation biomarkers but lower risk of CVD than men. Another study from Tampubolon and Maharani in 2018 among the older population found that AL score increased in sex difference. ${ }^{12}$ Compared with men, women showed an advantage in life expectancy. ${ }^{12}$ Taken together, these results suggest the use of sex-specific cutoff points for AL biomarkers in future research.

A number of studies have examined the association between AL and mortality among older adults. However, little attention has been paid to less developed regions, including China-the most populous country with the largest ageing population in the world. In 2019, there were 249 million adults aged 60 years or above in China, accounting for $17.3 \%$ of its total population, and this number is projected to almost double in 2050, reaching 487 million. ${ }^{13}{ }^{14}$ Understanding the relationship between $\mathrm{AL}$ and mortality in less developed countries is beneficial for leading to interventions, which could be helpful to change unhealthy lifestyles, decrease morbidity and mortality among the older population. In addition, less studies focus on sex-specific cut-off points for calculating AL index, this study will conduct sex-specific studies, which may closely reflect AL score among the older population.

In this study, we used a large cohort study to examine the association between AL and all-causes mortality among Chinese men and women aged at least 60 years. We hypothesised that a higher burden of AL would be associated with increased risk of all-cause mortality among both older men and women in China.

\section{METHODS}

\section{Data and study participants}

We used data from the Chinese Longitudinal Healthy Longevity Survey (CLHLS), an ongoing prospective, longitudinal study with the largest sample of the oldest old in China. Half of the counties and cities in 22 of the 31 provinces in China (covering $85 \%$ of the population) were randomly selected through a multistage cluster sampling approach. A wide range of sociodemographic, lifestyle, and health measures were collected in the CLHLS. The baseline survey was conducted in 1998 and participants who were alive were reinterviewed in each follow-up survey $(2000,2002,2005,2008-2009$, 2011-2012, 2014 and 2017-2018). In 2011-2012, an ancillary study, in which a blood test was added, was conducted in eight longevity areas: Laizhou City in Shandong Province, Xiayi County in Henan Province, Zhongxiang City in Hubei Province, Mayang County in Hunan Province, Yongfu County in Guangxi Autonomous Area, Sanshui District in Guangdong Province, Chengmai County in Hainan Province, and Rudong County in Jiangsu Province. All study participants gave informed consent. A more detailed description of the recruitment strategy and study design of the CLHLS has been published elsewhere. ${ }^{15-17}$ A total of 8959 individuals were included at baseline (1998). 1998 baseline survey, which was extended to
$11-162$ in 2000 , it was found that almost $30 \%$ died before 2002 interview and approximately $14 \%$ were lost that was higher than the attrition rate between 1998 and 2000 wave $(9.6 \%)$; the number of participants were extend to 16064 in 2002, and about 13.8\% were lost between 2002 and 2005; the number of interviewed were 15638 in 2005, and about $13.2 \%$ were lost between 2005 and 2008-2009; the number of participants were extended to 16.540 , and approximately $17.7 \%$ were lost between 2008-2009 and 2011-2012; the total number of interviewed participants were 9765 in $2011-2012 .^{18}$

A total of 2439 persons contributed blood samples in the ancillary study (2011-2012). Participants were excluded from the analytic sample if they had (1) incomplete data on any biomarkers for constructing AL $(n=251)$, (2) no follow-up data (time to death or censorship was undetermined; $n=552$ ), (3) had extreme values on the biomarkers $(n=109$; online supplemental table $S 1)$ or (4) were less than 60 years old $(n=16)$. The final analytical sample consisted of 1519 participants. We did not observe appreciable differences in age, ethnicity, marital status, smoking or chronic conditions between the analytical sample and those excluded $(n=920$; online supplemental table S2). Compared with the analytical sample, excluded persons had a higher education level and higher prevalence of exercise. In addition, compared with people who were lost follow-up, the included people had higher prevalence of married, and higher prevalence of stroke; we did not observe appreciable differences in age, ethnicity, smoking, exercise, hypertension, diabetes, heart disease, pulmonary, arthritis and cancer between both (online supplemental table S3).

\section{Calculation of AL score}

Based on previous research ${ }^{371920}$ and availability of data in the CLHLS, we selected nine biomarkers to construct AL: heart rate, systolic BP (SBP), and diastolic BP (DBP), body mass index (BMI), total cholesterol, high-density lipoprotein (HDL) cholesterol, glucose, triglyceride and $\mathrm{C}$ reactive protein (CRP). BMI, heart rate, SBP and DBP were collected from physical examinations. BMI was calculated as body weight (kilograms) divided by height (metres) squared. SBP and DBP were measured by a mercury sphygmomanometer with an appropriately sized cuff, taken in the seated position after 5 min of quiet rest under the supervision of trained research assistants. We used the average of two measurements for further analyses. Blood samples were used for assays of the level of the total cholesterol, HDL cholesterol, glucose, triglyceride and CRP.

To be in line with previous studies, ${ }^{4} 102122$ we used the highest quartile for heart rate, SBP, DBP, glucose and CRP and the lowest quartile for HDL cholesterol to define the high-risk group (coded 1). Because BMI, total cholesterol and triglyceride were inversely associated with mortality among older adults, especially the oldest old, ${ }^{23-25}$ we used the lowest quartile to define the highrisk group for these three biomarkers. For participants 
Table 1 Cut-point for each of nine biomarkers used to construct allostatic load

\begin{tabular}{|c|c|c|}
\hline \multirow[b]{2}{*}{ Biomarkers } & \multicolumn{2}{|l|}{ Cut-points } \\
\hline & Male & Female \\
\hline $\begin{array}{l}\text { Body mass index, } \\
\mathrm{kg} / \mathrm{m}^{2}\end{array}$ & $\leq 19.33 \mathrm{~kg} / \mathrm{m}^{2}$ & $\leq 17.78 \mathrm{~kg} / \mathrm{m}^{2}$ \\
\hline Glucose, $\mathrm{mmol} / \mathrm{L}$ & $\geq 5.13 \mathrm{mmol} / \mathrm{L}$ & $\geq 5.15 \mathrm{mmol} / \mathrm{L}$ \\
\hline $\begin{array}{l}\text { Total cholesterol, } \\
\mathrm{mmol} / \mathrm{L}\end{array}$ & $\leq 3.51 \mathrm{mmol} / \mathrm{L}$ & $\leq 3.71 \mathrm{mmol} / \mathrm{L}$ \\
\hline $\begin{array}{l}\text { High-density } \\
\text { lipoprotein } \\
\text { cholesterol, mmol/L }\end{array}$ & $\leq 1.04 \mathrm{mmol} / \mathrm{L}$ & $\leq 1.06 \mathrm{mmol} / \mathrm{L}$ \\
\hline Triglyceride, $\mathrm{mmol} / \mathrm{L}$ & $\leq 0.56 \mathrm{mmol} / \mathrm{L}$ & $\leq 0.63 \mathrm{mmol} / \mathrm{L}$ \\
\hline $\begin{array}{l}\text { High-sensitive } \mathrm{C} \\
\text { reactive protein, mg/L }\end{array}$ & $\geq 2.44 \mathrm{mg} / \mathrm{L}$ & $\geq 2.33 \mathrm{mg} / \mathrm{L}$ \\
\hline Heart rate, beats/min & $\geq 80$ beats $/ \mathrm{min}$ & $\geq 83$ beats $/ \mathrm{min}$ \\
\hline $\begin{array}{l}\text { Systolic blood } \\
\text { pressure, } \mathrm{mm} \mathrm{Hg}\end{array}$ & $\geq 150 \mathrm{~mm} \mathrm{Hg}$ & $\geq 160 \mathrm{~mm} \mathrm{Hg}$ \\
\hline $\begin{array}{l}\text { Diastolic blood } \\
\text { pressure, } \mathrm{mm} \mathrm{Hg}\end{array}$ & $\geq 90 \mathrm{~mm} \mathrm{Hg}$ & $\geq 90 \mathrm{~mm} \mathrm{Hg}$ \\
\hline
\end{tabular}

High-risk group was defined as below the sex-specific 25th percentile for body mass index, total cholesterol, high-density lipoprotein cholesterol and triglyceride. High-risk group was defined as above the sex-specific 75th percentile for glucose, high-sensitive $\mathrm{C}$ reactive protein, heart rate, systolic blood pressure and diastolic blood pressure.

who self-reported having been diagnosed with hypertension and heart disease, we classified their SBP, DBP and glucose into the high-risk category. Similarly, we classified participants' glucose into the high-risk group if they self-reported having been diagnosed with diabetes. The identification of risk quartiles of biomarkers is commonly used to construct AL index. ${ }^{526}$ The cut-points of all nine AL components by men and women were presented in table 1. We constructed the AL score based on the count of biomarkers falling in the high-risk group, ranging from 0 (lowest) to 9 (highest). To be in line with previous studies, ${ }^{27}$ we then considered using similar cut-off points, classifying the AL score into three categories based on sample distribution: 0-1 (low burden), 2-4 (medium burden) and 5-9 (high burden).

\section{Mortality}

The outcome was all-cause mortality. Vital status and date of death (for persons who died by the end of the study) was ascertained by the close family member or village doctor of the deceased participant during the follow-up survey in 2014 and 2017-2018. We calculated the survival time from the date of the baseline interview to the date of last interview (censored) or the death date.

\section{Covariates}

Demographic and lifestyle characteristics were collected by interview, including age, sex, ethnicity, education and marital status, smoking status and physical exercise. We divided ethnicity into Han and others (minority groups). Years of education were dichotomised as any (1 year or more) and no education, which is commonly way used in CLHLS study. ${ }^{28}{ }^{29}$ Marital status was dichotomised as married and others (widowed, not married and divorced). Cigarette smoking was categorised as current, past and non-smoker. Information of exercise was collected using the question 'Do you do exercise at present?' and dichotomised into yes or no. Chronic conditions were measured based on self-reported physician's diagnosis, including hypertension, diabetes, heart disease, stroke, pulmonary disease (including bronchitis, emphysema, pneumonia and asthma), arthritis and cancer.

\section{Statistical analyses}

All analyses were conducted separately for males and females. We first presented the relative frequency of the AL score using histograms and calculated the mean AL score. Then, we described the baseline characteristics of the study sample by AL burden (low, medium and high) using means and SDs for continuous variables and counts and percentages for categorical variables. Characteristics were compared across the three AL categories using analyses of variance for continuous variables and $\chi^{2}$ tests for categorical variables.

We calculated the death rates across three AL categories (low, medium and high burden). We used the Cox proportional hazards model to determine the unadjusted and adjusted associations between the AL and allcause mortality. Age, sex, education and marital status were included in the demographically adjusted models; smoking status, physical exercise and chronic status including pulmonary disease and arthritis were added in the fully adjusted models. We modelled AL both continuously and in categories.

Furthermore, one sensitivity analysis was undertaken, which aimed to exam if the model results were influenced when we did not use self-reported hypertension or diabetes to classify participants' risk category for $\mathrm{BP}$ or glucose.

All tests were two sided with a significance level of $p$ value less than 0.05 . We conducted all analyses using STATA V.16.0 (StataCorp).

\section{Study participants and public involvement}

This research was done without study participant involvement. Study participants were not invited to comment on the study design and were not consulted to develop participant-relevant outcomes or interpret the results. Participants were not invited to contribute to the writing or editing of this document for readability or accuracy.

\section{RESULTS}

\section{Distribution of AL categories}

The distribution of the AL score (range: 0-9) is rightskewed for both males and females; only $13(1.8 \%)$ males and $10(1.2 \%)$ females had a score of $6-9$, respectively 
The distribution of allostatic load score among male $(\mathrm{N}=709)$

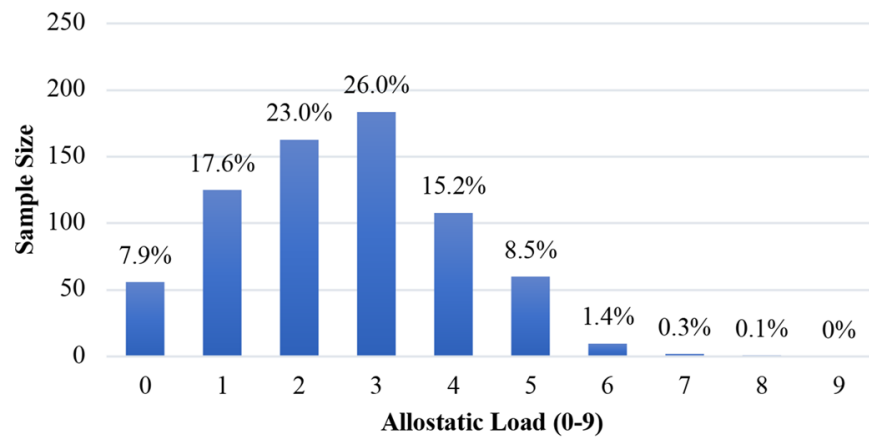

The distribution of allostatic load score among female $(\mathbf{N}=\mathbf{8 1 0})$

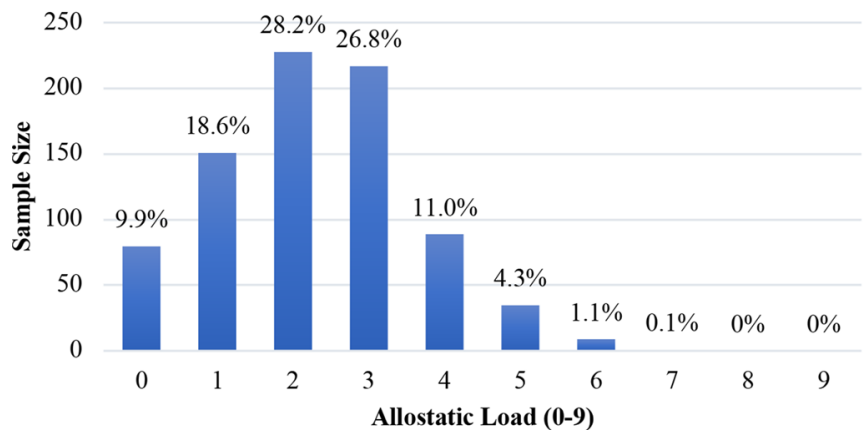

Figure 1 Distribution of sample size by allostatic load (AL) score among males and females. The above figure is the distribution of $A L$ score among male. The below figure is the distribution of $A L$ score among female. The distribution of the AL score (range: $0-9$ ) is rightskewed for both males and females; only 13 (1.8\%) males and $10(1.2 \%)$ females had a score of $6-9$, respectively.

(figure 1). The mean AL score was $2.56(\mathrm{SD}=1.47)$ for males and $2.28(\mathrm{SD}=1.34)$ for females. For males, $25.5 \%$, $64.2 \%$, and $10.3 \%$ had an AL score of $0-1,2-4$, and 5-9, respectively. For females, $28.5 \%, 65.9 \%$ and $5.6 \%$ had an AL score of 0-1 (low burden), 2-4 (medium burden) and 5-9 (high burden), respectively.

\section{Demographic characteristics}

A total of $709(46.7 \%)$ males were included. The average age for males with an AL score of 0-1 (low burden), 2-4 (medium burden), 5-9 (high burden) was 77.6, 81.0 and 84.1 years, respectively $(\mathrm{p}=0.042)$. In addition, we observed significant differences in the prevalence hypertension and diabetes by different AL burden among males.

The study sample included $810(53.3 \%)$ females. The average age for females with an AL score of 0-1 (low burden), 2-4 (medium burden), 5-9 (high burden) was 87.0, 91.3 and 93.6 years, respectively $(p<0.05$; table 2$)$. Compared with men, women were older, less educated, had a lower prevalence of smoking, and were less physically active. Females with a lower AL were more likely to be married and have any education than woman with higher AL score; they also had a lower prevalence of hypertension, diabetes and heart disease. We did not observe significant difference in ethnicity, smoking, physical exercise, stroke, pulmonary disease, arthritis and cancer across AL burden (low, medium and high) among females.

\section{Association between AL and mortality among males}

A total of 310 males died; the overall death rate was 105.7 per 1000 person-years. Males with an AL score of 0-1 (low), 2-4 (medium) and 5-9 (high) had a death rate of $66.1,110.4 .6$ and 201.3 per 1000 person-years, respectively (table 3 ).

In the unadjusted Cox model, per unit higher AL score was significantly associated with a $75 \%$ higher hazard of death among males (95\% CI $44 \%$ to $112 \%$; table 3 ). The association slightly attenuated but persisted in the full adjusted model (HR 1.51, 95\% CI 1.23 to 1.84 ). When modelled in categories, in the unadjusted model, the hazard of death of male with the medium AL burden (score: 2-4) was 1.68 times than hazard of death of those with a lower AL burden (score: 0-1); Male with a high AL burden (Score: 5-9) had a more than threefold higher hazard of death than those with a lower AL burden (score: 0-1). These associations persisted after adjustment of sociodemographics and lifestyles. In the fully adjusted model, males with a medium AL burden (score: 2-4) had a $33 \%$ higher hazard of death than those with a low AL burden (score: 0-1). Males with a high AL burden (score: 5-9) had a more than twofold higher hazard of death than those with a low AL burden (score: 0-1). Results did not change substantially in the sensitivity analyses (online supplemental table $\mathrm{S} 4$ ).

\section{Association between AL and mortality among females}

Over an average follow-up period of 3.9 years, 787 deaths (51.8\%) occurred. A total of 477 females died $(58.9 \%)$; the overall death rate was 161.3 per 1000 person-years. The death rates for females with an AL score of 0-1 (low burden), 2-4 (medium burden) and 5-9 (high burden) were 121.1, 174.8, and 252.6 per 1000 person-years, respectively (table 3 ).

In the unadjusted Cox model, per unit higher AL (modelled continuously) was significantly associated with a $45 \%$ higher hazard of death among females (95\% CI $23 \%$ to $72 \%$; table 3 ). However, the association attenuated and became insignificant after adjusting for sociodemographics (age, sex, ethnicity, education and marital status); similar results were observed when smoking, exercise and chronic conditions including pulmonary disease and arthritis were additionally adjusted (HR 1.16, 95\% CI 0.97 to 1.38$)$. When modelled in categories, the HR was 1.44 (95\% CI 1.17 to 1.79$)$ and 2.11 (95\% CI 1.44 to 3.10) for females with an AL score of 2-4 and 5-9, respectively, compared with those with a score of $0-1$ in the unadjusted model. After multivariable adjustment, females with an AL score of 2-4 and 5-9 had a 11\% and $34 \%$ higher hazard of death, although the associations 


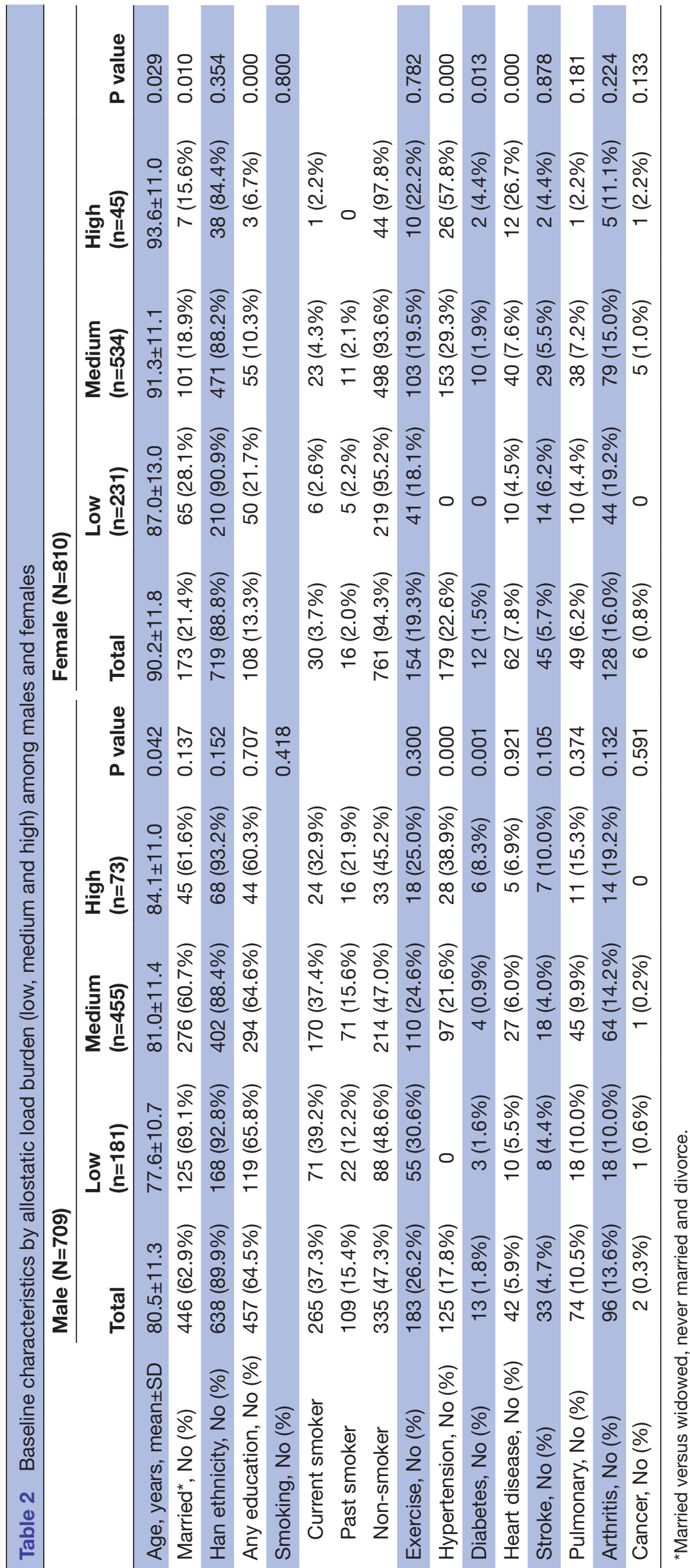


Table 3 Association between allostatic load and mortality among males and females

Males ( $\mathbf{N}=709)$

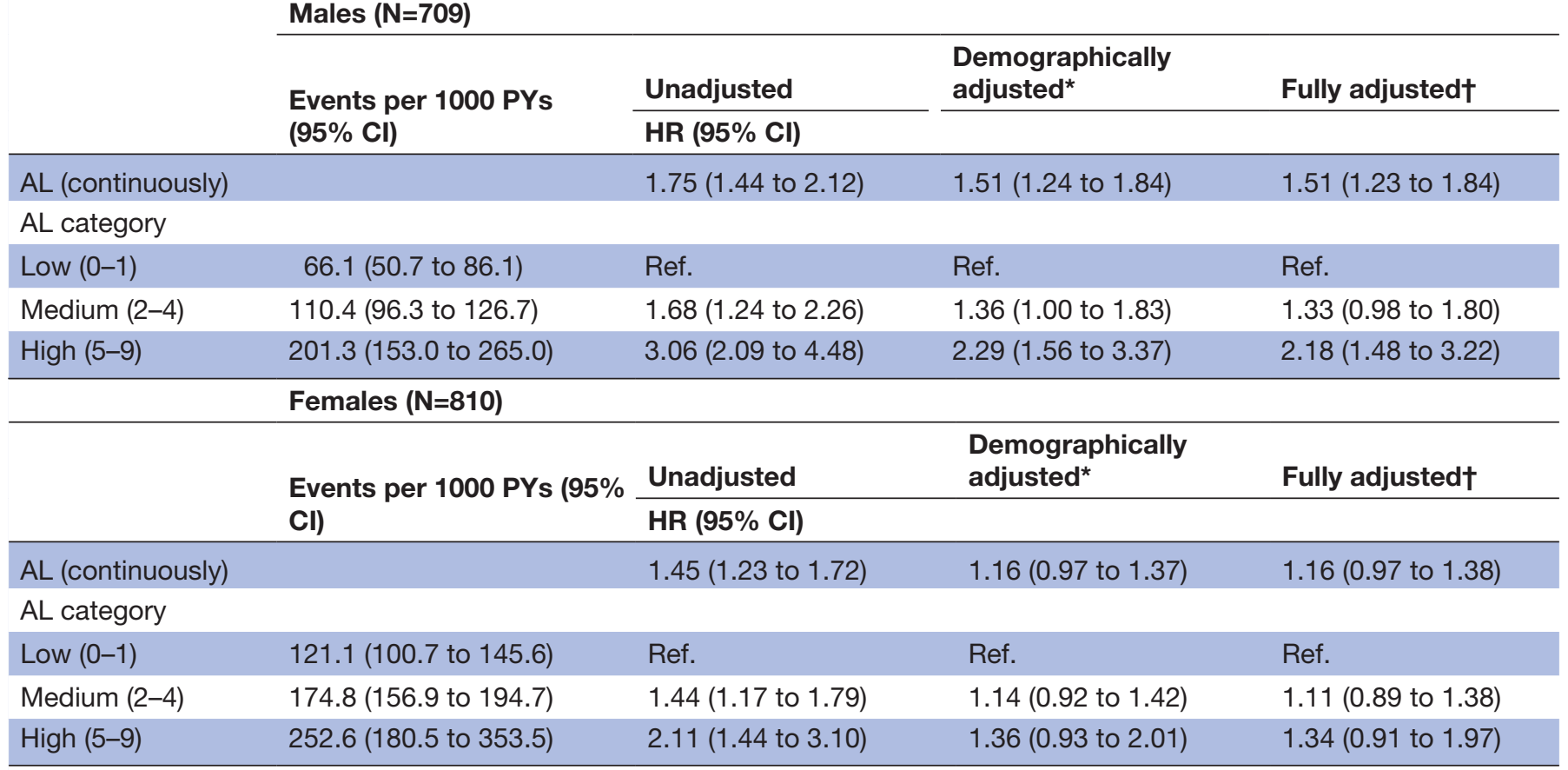

*Demographically adjusted model included age, ethnicity (Han vs minority), education (any vs none), and marital status (married vs others). †Fully adjusted model included age, ethnicity (Han vs minority), education (any vs none) and marital status (married vs others), smoking (current vs previous or non-smoke), exercise (yes vs no) and chronic diseases such as pulmonary disease and arthritis.

$\mathrm{AL}$, allostatic load; PY, person-year.

were not significant. Results did not change substantially in the sensitivity analyses (online supplemental table S4).

\section{DISCUSSION}

The present study aimed to explore the association between AL burden and all-cause mortality among men and women aged at least 60 years in China. This finding is somewhat consistent with previous evidence suggesting that $\mathrm{AL}$ may be predictor of all-cause mortality later in life. ${ }^{719}$ In addition, we found that older men with high AL burden had a more than twofold hazard of death than those with a low AL burden. There is no significant association observing among females, but the findings are trending in the expected direction. These findings were in line with previous studies showing men tend to have higher AL with higher risk of death than women, and gender difference among AL score and cause-specific mortality risk including infectious diseases, cardiometabolic disease and malignant neoplasm. ${ }^{73031}$ One possible explanation for the sex differences in the association between AL and mortality among older adults is that older women might be less vulnerable to stress men due to sex differences in the hippocampal formation in humans. ${ }^{32-34}$ In addition, it has been shown that oestrogen plays an important role in the brain with the development of ageing, aiming to maintain allostasis when facing physiological stress, which may be possible to protect women against age-related diseases. ${ }^{34-36}$ Moreover, Gruenewald $e t$ $a l^{37}$ stated that gender difference in forecasting mortality risk among older people by biomarkers; compared with female, neuroendocrine and immune-related biomarkers were more predictive in males. ${ }^{37}$ In addition, the sex difference in the association between AL and mortality may be explained by behavioural factors. Social support is effective in relieving stress. ${ }^{38}$ Women are more socially active to seek emotional support when facing stress than men. ${ }^{38}$ Furthermore, in our study, women had high mean age, less education, lower prevalence of smoking and less exercise. Therefore, adjustment of these covariates may influence the significant level of association in the final model.

To our knowledge, our study was the first to reveal a significant association between AL and mortality among male participants only. However, we need to interpret these results with caution because the findings regarding the association between $\mathrm{AL}$ and mortality among females were trending in the expected direction. A study with more female participants is needed to provide a more definite conclusion. Previous study has identified increased risk of all-cause mortality associated with increasing AL score in men. ${ }^{7}$ We found that the strength of the association differed between the present study and Hwang $e$ t $a l$ s work among men. There are several plausible explanations for this discrepancy. First, the population in Hwang et al's study ( $\geq 54$ years) was younger than ours ( $\geq 60$ years). Second, we did not include cortisol, which is a commonly used indicator of the primary mediator stress, due to data unavailability. Surrogate measures 
were used in the present study, which may lead to weaker associations. Third, two studies used different cut-points for constructing the AL score. We used sex-specific cut-off points for each biomarker, while general cut-points were used in Hwang $e t a l$ s work. Fourth, follow-up length was different between the two studies. Furthermore, different choices of model covariates may influence the strength of observed associations. Hwang et als research only adjusted for age and sex.

For the sensitivity analysis of the association between AL and mortality, the overall magnitude of the HR was not largely altered, and the association was still statically significant. This suggests that the results of the association between AL category and mortality may not influenced by the participants who were self-reported disease status, which may support that our results are robust (online supplemental table S4).

The AL was initially constructed using primary mediator stress including cortisol, epinephrine and norepinephrine. These biomarkers are not widely available and secondary responses of cardiovascular, inflammatory and metabolic biomarkers such as CRP, BP and heart rate were used as surrogate measures. Although multiple CVD risk factors were included, AL, in theory, represents multisystem physiological dysregulation instead of functional decline in one system. Previous studies showed that AL was able to stratify the risk of a wider range of health outcomes than traditional CVD risk factors, ${ }^{4}$ it appears that AL could predict more health information including CVD incident, decline of cognition function, decline of physical function and mortality than traditional CVD risk factors. The current study did not have data on primary mediator of AL, so we did not conduct separate analysis to investigate the association between primary vs secondary mediators of AL and mortality.

This study has some strengths. This is among the first to investigate the association between $\mathrm{AL}$ and mortality using a Chinese population. Moreover, our study used of CLHLS dataset that is a large nationally representative old population survey in China. Furthermore, we updated the quartile risk method for biomarkers BMI, total cholesterol and triglyceride due to inversely association with mortality among older adults, which may more truly reflect AL score in old people. Additionally, our study added evidence to support sex differences in the association between $\mathrm{AL}$ and the increased risk of mortality. Finally, sex-specific cut-off points were used to construct the AL score may more truly reflect association between AL and mortality in different gender compared with previous study. Additionally, we collected detailed covariates information including age, sex, marital status, ethnicity, education, chronic diseases, which enable us to adjust for a range of potential confounders in the final cox model.

Despite these strengths, we acknowledge some limitations. First, we did not include any primary neuroendocrine biomarkers such as cortisol in constructing the AL score due to data unavailability; this might partially explain the null finding regarding the association between AL and mortality among women. The cortisol biomarker plays an important role in responding stress, which needs repeated measurements within 1-2 days which causes difficulties to measure in large national survey. ${ }^{39}$ Inclusion of cortisol biomarker maybe improve our power of AL score predictions for mortality. Additionally, we classified AL biomarkers based on sex-specific quartiles; however, these measures may vary over time, leading to misclassification. Furthermore, there is huge lost to follow-up (>20\%; 552 of 2439), although only 3-7 year range of follow-up (2011 to 2014-2018), which may underestimate the association. Then, participants who provided blood sample in this study were residents in eight longevity areas including Laizhou of Shandong Province, Xiayi of Henan Province, Zhongxiang of Hubei Province, Mayang of Hunan Province, Sanshui of Guangdong Province, Yongfu of Guangxi Autonomous Region, Chengmai of Hainan Province, Rudong of Jiangsu Province, which were from 8 of 23 provinces, five autonomous regions, four municipalities and two special administrative regions. Therefore, our results may not be greatly generalisable to older adults living in other regions of China. Lastly, it is important to notice that the sample was extremely old, which may influence the magnitude of the association presented.

Therefore, even though we found that higher AL burden was associated with increased all-cause mortality among Chinese men aged at least 60 years, but not women, it is really recommended that these results need to be replicated in large longitudinal studies with longer follow-up time, with more AL biomarkers such as cortisol, or with containing Chinese from more regions apart from eight longevity areas. This would helpful for comparing with our results and validating them in different populations.

\section{CONCLUSION}

In conclusion, our study showed that higher AL burden was associated with increased all-cause mortality among Chinese men aged at least 60 years. We did not find strong evidence among women. Intervention programmes targeting modifiable components of the AL burden may help prolong lifespan for older adults, especially men, in China.

Contributors THZ conceived of and conducted the data analyses, interpreted the findings and wrote the manuscript. LJY, H-SC and H-YJ contributed to manuscript revision. CW contributed to the analysis and interpretation of data and drafting and revision of the manuscript.

Funding The research results of this article are sponsored partly by the Kunshan Municipal Government research funding.

Disclaimer The funder played no role in study design, data collection, and analysis, the decision to publish, or preparation of the manuscript.

Competing interests None declared.

Patient consent for publication Not required.

Ethics approval The original CHARLS was approved by the Ethical Review Committee of Peking University. The baseline data collection was obtained from the 
Biomedical Ethics Review Committee of Peking University (IRB00001052-11015), and all participants have signed informed consent.

Provenance and peer review Not commissioned; externally peer reviewed.

Data availability statement Data are available in a public, open access repository. The raw data are available on website: https://opendata.pku.edu.cn/dataset.xhtml? persistentld=doi:10.18170/DVN/WB07LK.

Supplemental material This content has been supplied by the author(s). It has not been vetted by BMJ Publishing Group Limited (BMJ) and may not have been peer-reviewed. Any opinions or recommendations discussed are solely those of the author(s) and are not endorsed by BMJ. BMJ disclaims all liability and responsibility arising from any reliance placed on the content. Where the content includes any translated material, BMJ does not warrant the accuracy and reliability of the translations (including but not limited to local regulations, clinical guidelines, terminology, drug names and drug dosages), and is not responsible for any error and/or omissions arising from translation and adaptation or otherwise.

Open access This is an open access article distributed in accordance with the Creative Commons Attribution Non Commercial (CC BY-NC 4.0) license, which permits others to distribute, remix, adapt, build upon this work non-commercially, and license their derivative works on different terms, provided the original work is properly cited, appropriate credit is given, any changes made indicated, and the use is non-commercial. See: http://creativecommons.org/licenses/by-nc/4.0/.

ORCID iD

Chenkai Wu http://orcid.org/0000-0002-2256-0653

\section{REFERENCES}

1 Beckie TM. A systematic review of allostatic load, health, and health disparities. Biol Res Nurs 2012;14:311-46.

2 Karlamangla AS, Singer BH, Seeman TE. Reduction in allostatic load in older adults is associated with lower all-cause mortality risk: MacArthur studies of successful aging. Psychosom Med 2006;68:500-7.

3 Robertson T, Beveridge G, Bromley C. Allostatic load as a predictor of all-cause and cause-specific mortality in the general population: evidence from the Scottish health survey. PLoS One 2017;12:e0183297.

4 Seeman TE, McEwen BS, Rowe JW, et al. Allostatic load as a marker of cumulative biological risk: MacArthur studies of successful aging. Proc Natl Acad Sci U S A 2001;98:4770-5.

5 Juster R-P, McEwen BS, Lupien SJ. Allostatic load biomarkers of chronic stress and impact on health and cognition. Neurosci Biobehav Rev 2010;35:2-16.

6 Gruenewald TL, Seeman TE, Karlamangla AS, et al. Allostatic load and frailty in older adults. J Am Geriatr Soc 2009;57:1525-31.

7 Hwang A-C, Peng L-N, Wen Y-W, et al. Predicting all-cause and cause-specific mortality by static and dynamic measurements of allostatic load: a 10-year population-based cohort study in Taiwan. J Am Med Dir Assoc 2014;15:490-6.

8 Karlamangla AS, Singer BH, McEwen BS, et al. Allostatic load as a predictor of functional decline. MacArthur studies of successful aging. J Clin Epidemiol 2002;55:696-710.

9 Levine ME, Crimmins EM. A comparison of methods for assessing mortality risk. Am J Hum Biol 2014;26:768-76.

10 Seeman T, Gruenewald T, Karlamangla A, et al. Modeling multisystem biological risk in young adults: the coronary artery risk development in young adults study. Am J Hum Biol 2010;22:463-72.

11 Yang Y, Kozloski M. Sex differences in age trajectories of physiological dysregulation: inflammation, metabolic syndrome, and allostatic load. J Gerontol A Biol Sci Med Sci 2011;66:493-500.

12 Tampubolon G, Maharani A. Trajectories of allostatic load among older Americans and Britons: longitudinal cohort studies. BMC Geriatr 2018;18:255

13 China population (age 60+): the 2019 revision, 2019. Available: https://population.un.org/wpp/Graphs/Probabilistic/POP/60plus/156

14 Worldometers. China population, 2019. Available: https://www. worldometers.info/world-population/china-population
15 Gu D, Dupre ME. Assessment of reliability of mortality and morbidity in the 1998-2002 CLHLS waves. In: Healthy longevity in China. Dordrecht: Springer, 2008: 99-116.

$16 \mathrm{Yi}$ Z. Introduction to the chinese longitudinal healthy longevity survey (CLHLS). In: Healthy longevity in China. Dordrecht: Springer, 2008: 23-38.

$17 \mathrm{Yi}$ Z. Reliability of age reporting among the Chinese oldest-old in the CLHLS datasets. In: Healthy longevity in China. Dordrecht: Springer, 2008: 61-78.

18 Zeng Y, Poston DL, Vlosky DA, eds. Healthy longevity in China: demographic, socioeconomic, and psychological dimensions. Springer Science \& Business Media, 2008.

19 Borrell LN, Dallo FJ, Nguyen N. Racial/Ethnic disparities in all-cause mortality in U.S. adults: the effect of allostatic load. Public Health Rep 2010;125:810-6.

20 Geronimus AT, Hicken M, Keene D, et al. "Weathering" and age patterns of allostatic load scores among blacks and whites in the United States. Am J Public Health 2006;96:826-33.

21 Crimmins EM, Johnston M, Hayward M, et al. Age differences in allostatic load: an index of physiological dysregulation. Exp Gerontol 2003;38:731-4.

22 Duong MT, Bingham BA, Aldana PC, et al. Variation in the calculation of allostatic load score: 21 examples from NHANES. J Racial Ethn Health Disparities 2017;4:455-61.

23 Lv Y-B, Mao C, Gao X. Triglycerides paradox among the oldest Old: "the lower the better?". J Am Geriatr Soc 2019;67:741-8.

24 Winter JE, Maclnnis RJ, Wattanapenpaiboon N, et al. BMI and all-cause mortality in older adults: a meta-analysis. Am J Clin Nutr 2014:99:875-90.

25 Weverling-Rijnsburger AW, Blauw GJ, Lagaay AM, et al. Total cholesterol and risk of mortality in the oldest old. Lancet 1997;350:1119-23.

26 Read S, Grundy E. Allostatic load and health in the older population of England: a crossed-lagged analysis. Psychosom Med 2014;76:490.

27 Castagné R, Garès V, Karimi M, et al. Allostatic load and subsequent all-cause mortality: which biological markers drive the relationship? findings from a UK birth cohort. Eur J Epidemiol 2018;33:441-58.

28 Zhu A, Yan LL, Wu C-D, et al. Residential greenness, activities of daily living, and instrumental activities of daily living: a longitudinal cohort study of older adults in China. Environ Epidemiol 2019;3:e065

29 J S J, Zhu A, Bai C, et al. Residential greenness and mortality in oldest-old women and men in China: a longitudinal cohort study. The Lancet

30 Seeman TE, Singer BH, Ryff CD, et al. Social relationships, gender, and allostatic load across two age cohorts. Psychosom Med 2002;64:395-406.

31 Thomson EM, Kalayci H, Walker M. Cumulative toll of exposure to stressors in Canadians: an allostatic load profile. Health Rep 2019;30:14-21.

32 Gould E, Westlind-Danielsson A, Frankfurt M, et al. Sex differences and thyroid hormone sensitivity of hippocampal pyramidal cells. J. Neurosci. 1990;10:996-1003.

33 Kimura D. Sex differences in the brain. Sci Am 1992:267:118-25.

34 Paulson PE, Minoshima S, Morrow TJ, et al. Gender differences in pain perception and patterns of cerebral activation during noxious heat stimulation in humans. Pain 1998;76:223-9.

35 Borrás C, Gambini J, Carmen Gómez-Cabrera M, et al. 17ßoestradiol up-regulates longevity-related, antioxidant enzyme expression via the ERK1 and ERK2. Aging Cell 2005;4:113-8.

36 Viña J, Borrás C, Gambini J, et al. Why females live longer than males: control of longevity by sex hormones. Sci Aging Knowledge Environ 2005;2005:pe17.

37 Gruenewald TL, Seeman TE, Ryff CD, et al. Combinations of biomarkers predictive of later life mortality. Proc Natl Acad Sci U S A 2006;103:14158-63.

38 Hamaideh SH. Gender differences in stressors and reactions to stressors among Jordanian university students. Int J Soc Psychiatry 2012;58:26-33

39 Dowd JB, Simanek AM, Aiello AE. Socio-Economic status, cortisol and allostatic load: a review of the literature. Int $\mathrm{J}$ Epidemiol 2009;38:1297-309. 